\title{
A New Method for Indirect Mass Measurements using the Integral Charge Asymmetry at the LHC
}

\author{
Steve Muanza* \\ CPPM Marseille, CNRS/IN2P3 \& Aix-Marseille University \\ E-mail: muanza@in2p3.fr
}

\begin{abstract}
We propose a novel method for an indirect measurement of the mass of final states produced through charged current processes at the LHC. This method is based upon the process integral charge asymmetry. First, the theoretical prediction of the integral charge asymmetry and its related uncertainties are studied through parton level cross sections calculations. Then, the experimental extraction of the integral charge asymmetry of a given signal, in the presence of some background, is performed using particle level simulations. Process dependent templates enable to convert the measured integral charge asymmetry into an estimated mass of the charged final state. Finally, a combination of the experimental and the theoretical uncertainties determines the full uncertainty of the indirect mass measurement.

This new method applies to all charged current processes at the LHC. In this study, we demonstrate its effectiveness at extracting the mass of the $\mathrm{W}$ boson, as a first step, and the sum of the masses of a chargino and a neutralino in case these supersymmetric particles are produced by pair, as a second step. Note that contrarily to most of the usual mass reconstruction techniques that are based upon the kinematics of the events final state, this method depends on the events initial state and reflects the charge asymmetry of the colliding protons.
\end{abstract}

The XXIII International Workshop on Deep Inelastic Scattering and Related Subjects

April 27 - May 1, 2015

Southern Methodist University

Dallas, Texas 75275

${ }^{*}$ Speaker. 


\section{Introduction}

Contrarily to most of the previous high energy particle colliders, the LHC is a charge asymmetric machine. For charged final states ${ }^{1}$, denoted $F S^{ \pm}$, the integral charge asymmetry, denoted $A_{C}$, is defined by

$$
A_{C}=\frac{N\left(F S^{+}\right)-N\left(F S^{-}\right)}{N\left(F S^{+}\right)+N\left(F S^{-}\right)}
$$

where $N\left(F S^{+}\right)$and $N\left(F S^{-}\right)$represent respectively the number of events bearing a positive and a negative charge in the FS.

For a $F S^{ \pm}$produced at the LHC in $p+p$ collisions, this quantity is positive or null, whilst it is always compatible with zero for a $F S^{ \pm}$produced at the TEVATRON in $p+\bar{p}$ collisions.

To illustrate the $A_{C}$ observable, let's consider the Drell-Yan production of $W^{ \pm}$bosons in $p+p$ collisions. It is obvious for this simple $2 \rightarrow 2$ s-channel process that more $W^{+}$than $W^{-}$are produced. Indeed, denoting $y_{W}$ the rapidity of the $\mathrm{W}$ boson, the corresponding range of the Björken x's: $x_{1,2}=\frac{M_{W^{ \pm}}}{\sqrt{s}} \times e^{ \pm y_{W}}$, probes the charge asymmetric valence parton densities within the proton. This results in having more $U+\bar{D} \rightarrow W^{+}$than $\bar{U}+D \rightarrow W^{-}$configurations in the initial state (IS). Here $\mathrm{U}$ and $\mathrm{D}$ collectively and respectively represent the up and the down quarks.

In the latter case the dominant contribution to $A_{C}$ comes from the difference in rate between the $u+\bar{d}$ and the $d+\bar{u}$ quark currents in the IS. Using the usual notation $f\left(x, Q^{2}\right)$ for the parton density functions (PDF) and within the leading order (LO) approximation, this can be expressed as:

$$
A_{C} \approx \frac{u\left(x_{1,2}, M_{W}^{2}\right) \bar{d}\left(x_{2,1}, M_{W}^{2}\right)-\bar{u}\left(x_{1,2}, M_{W}^{2}\right) d\left(x_{2,1}, M_{W}^{2}\right)}{u\left(x_{1,2}, M_{W}^{2}\right) \bar{d}\left(x_{2,1}, M_{W}^{2}\right)+\bar{u}\left(x_{1,2}, M_{W}^{2}\right) d\left(x_{2,1}, M_{W}^{2}\right)}
$$

where the squared four-momentum transfer $Q^{2}$ is set to $M_{W}^{2}$.

From equation 1.2, we can see that the $Q^{2}$ evolution of the parton density functions (PDFs) govern the $Q^{2}$ evolution of $A_{C}$. The former are known, up-to the NNLO in QCD, as solutions of the DGLAP equations [2]. One could therefore think of using an analytical functional form to relate $A_{C}$ to the squared mass of the s-channel propagator, here $M_{W}^{2}$. However there are additional contributions to the $W^{ \pm}$inclusive production. At the Born level, some come from other flavour combinations in the IS of the s-channel, and some come from the $\mathrm{u}$ and the t-channels. On top of this, there are higher order corrections. These extra contributions render the analytical expression of the $Q^{2}$ dependence of $A_{C}$ much more complicated. Therefore we choose to build process-dependent numerical mass template curves for $A_{C}$ by varying $\mathrm{M}_{F S^{ \pm}}$. These mass templates constitute inclusive and flexible tools into which all the above-mentioned contributions to $A_{C}$ can be incorporated, they can very easily be built within restricted domain of the signal phase space imposed by kinematic cuts.

In this article we exploit the $A_{C}$ to set a new type of constraint on the mass of the charged $F S^{ \pm}$ as initially proposed in [8][9].

We'll separate the study into two parts. The first one, in section 2, is dedicated to present in full length the method of indirect mass measurement that we propose on a known Standard Model

\footnotetext{
${ }^{1}$ We defined these as event topologies containing an odd number of high $p_{T}$ charged and isolated leptons within the fiducial volume of the detector.
} 
(SM) process. We choose the $W^{ \pm} \rightarrow \ell^{ \pm}+\mathbb{E}_{T}$ inclusive production at the LHC to serve as a test bench.

In the second part, in section 3, we shall repeat the method on a "Beyond the Standard Model" (BSM) process. We choose a SUSY search process of high interest, namely $\tilde{\chi}_{1}^{ \pm}+\tilde{\chi}_{2}^{0} \rightarrow 3 \ell^{ \pm}+\mathbb{E}_{T}$. For both the SM and the BSM processes, we obviously tag the sign of the FS by choosing a decay into one (or three) charged lepton(s) for which the sign is experimentally easily accessible.

\section{A Standard Model Test Bench Process}

\subsection{Theoretical Prediction of $A_{C}\left(W^{ \pm} \rightarrow \ell^{ \pm} v\right)$}

The theoretical $A_{C}$ template curves for the MRST2007lomod PDF. The raw template curve with the breakdown of the theory uncertainties and the fit using a functional form described below are shown on the left hand side (LHS) and the right hand side (RHS) of Fig. 1, respectively. We note the evolution of $A_{C}$ as a function of the artificially varied $W^{ \pm}$is a monotonic increasing function that can be described by a polynomial of logarithms of logarithms of $W^{ \pm}: A_{C}\left[M_{W^{ \pm}}\right]=$ $\sum_{i=0}^{N} A_{i} \times\left[\log \left[\log \left[M_{W^{ \pm}}\right]\right]\right]^{i}$. This functional form is inspired by a parametrization of the analytical solution of non-singlet DGLAP equation.
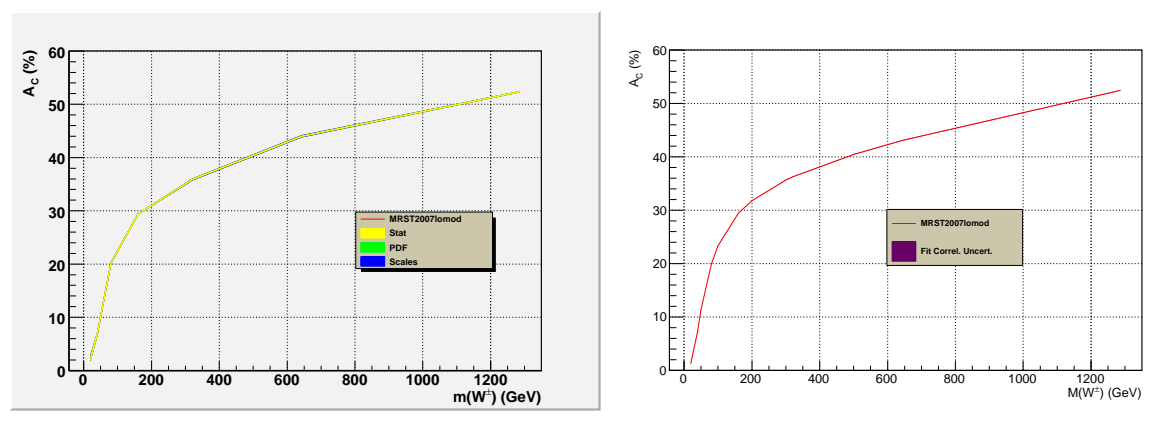

Figure 1: Theoretical $A_{C}$ template curves for the MRST2007lomod PDF.

\subsection{Experimental Measurement of $A_{C}\left(W^{ \pm} \rightarrow \ell^{ \pm} v\right)$}

In this subsection we extract $A_{C}\left(W^{ \pm} \rightarrow \ell^{ \pm} v\right)$ in presence of some background processes. To this end we generate Monte Carlo samples for the signal and the background processes in $\mathrm{p}+\mathrm{p}$ colissions at $\sqrt{s}=7 \mathrm{TeV}$ with an integrated luminosity of $L=1 \mathrm{fb}^{-1}$. We use a fast simulation of the response of the ATLAS detector. And we apply some selection cuts to optimize the signal to noise ratio. We keep the events with a hard and isolated electron or muon that has a $p_{T}>25$ $\mathrm{GeV}$. These events must have a missing transverse energy larger than $25 \mathrm{GeV}$ and a transverse mass larger than $40 \mathrm{GeV}$.

The corresponding efficiencies, events yields and expected charge asymmetries reported in Tab. 1. In order to correct for the experimenal biases on $A_{C}$, one due to the selection cuts and the other due to events from background processes passing these selection cuts, we apply an $A_{C}$ background subtraction. This method background subtraction is based upon the following equation: 


\begin{tabular}{|c|c|c|c|}
\hline Process & $\begin{array}{c}\varepsilon \\
(\%)\end{array}$ & $\begin{array}{c}N_{\exp } \\
(\mathrm{k} \text { evts })\end{array}$ & $\begin{array}{c}A_{C} \pm \delta A_{C}^{\text {SIat }} \\
(\%)\end{array}$ \\
\hline \hline Signal: $W^{ \pm} \rightarrow e^{ \pm} v_{e}$ & & & \\
$M\left(W^{ \pm}\right)=40.2 \mathrm{GeV}$ & $0.81 \pm 0.01$ & 290.367 & $9.66 \pm 1.57$ \\
$M\left(W^{ \pm}\right)=60.3 \mathrm{GeV}$ & $13.69 \pm 0.05$ & 2561.508 & $11.22 \pm 0.38$ \\
$M\left(W^{ \pm}\right)=\underline{80.4 \mathrm{GeV}}$ & $29.59 \pm 0.04$ & 3343.195 & $16.70 \pm 0.18$ \\
$M\left(W^{ \pm}\right)=100.5 \mathrm{GeV}$ & $39.19 \pm 0.07$ & 2926.093 & $20.77 \pm 0.22$ \\
$M\left(W^{ \pm}\right)=120.6 \mathrm{GeV}$ & $44.84 \pm 0.07$ & 2357.557 & $23.19 \pm 0.21$ \\
$M\left(W^{ \pm}\right)=140.7 \mathrm{GeV}$ & $48.66 \pm 0.07$ & 1899.820 & $25.29 \pm 0.20$ \\
$M\left(W^{ \pm}\right)=160.8 \mathrm{GeV}$ & $51.28 \pm 0.07$ & 1527.360 & $26.87 \pm 0.19$ \\
$M\left(W^{ \pm}\right)=201.0 \mathrm{GeV}$ & $54.54 \pm 0.07$ & 1.032 & $29.06 \pm 0.18$ \\
\hline \hline Background & - & $91.614 \pm 1.706$ & $10.07 \pm 0.15$ \\
\hline$W^{ \pm} \rightarrow \mu^{ \pm} v_{\mu} / \tau^{ \pm} v_{\tau} / q \bar{q}^{\prime}$ & $0.211 \pm 0.003$ & 71.350 & $12.92 \pm 1.25$ \\
\hline$t \bar{t}$ & $5.76 \pm 0.02$ & 6.600 & $1.00 \pm 0.37$ \\
$t+b, t+q(+b)$ & $3.59 \pm 0.01$ & 1.926 & $28.97 \pm 0.35$ \\
\hline$W+W, W+\gamma^{*} / Z, \gamma^{*} / Z+\gamma^{*} / Z$ & $2.94 \pm 0.01$ & 2.331 & $10.65 \pm 0.35$ \\
\hline$\gamma+\gamma, \gamma+j e t s, \gamma+W^{ \pm}, \gamma+Z$ & $0.201 \pm 0.001$ & 0.759 & $17.25 \pm 0.53$ \\
\hline$\gamma^{*} / Z$ & $0.535 \pm 0.001$ & 5.746 & $4.43 \pm 0.23$ \\
\hline $\mathrm{QCD} \mathrm{HF}$ & $(0.44 \pm 0.17) \times 10^{-4}$ & 1.347 & $14.29 \pm 37.41$ \\
$\mathrm{QCD} \mathrm{LF}$ & $(0.87 \pm 0.33) \times 10^{-4}$ & 1.555 & $71.43 \pm 26.45$ \\
\hline
\end{tabular}

Table 1: Selection efficiencies, event yields and integral charge asymmetries for the $W^{ \pm} \rightarrow e^{ \pm} v_{e}$ analysis.

$A_{C}^{E x p}(S)=\left(1+\alpha^{E x p}\right) \cdot A_{C}^{E x p}(S+B)-\alpha^{E x p} \cdot A_{C}^{E x p}(B)$ where $\alpha^{E x p}=\frac{N_{B}^{E x p}}{N_{S}^{E x p}}$ is the noise (B) to signal (S) ratio. We also use this equation to propagate the experimental systematic uncertainties into $A_{C}^{E x p}(S)$ accounting for the correlations between $\alpha^{E x p}, A_{C}^{E x p}(B)$, and $A_{C}^{E x p}(S+B)$.

This procedure enables to obtain $A_{C}^{\text {Meas }}(S)$ starting from $A_{C}^{E x p}(S)$ where "Meas" stands for measured and represents $A_{C}(S)$ after the background subtraction and the propagation of the experimental systematic uncertainties. The evolution of $A_{C}^{\text {Meas }}(S)$ with respect to $M_{W^{ \pm}}$enables to construct experimental $A_{C}$ template curves. This is illustrated for the electron channel in Fig. 2.
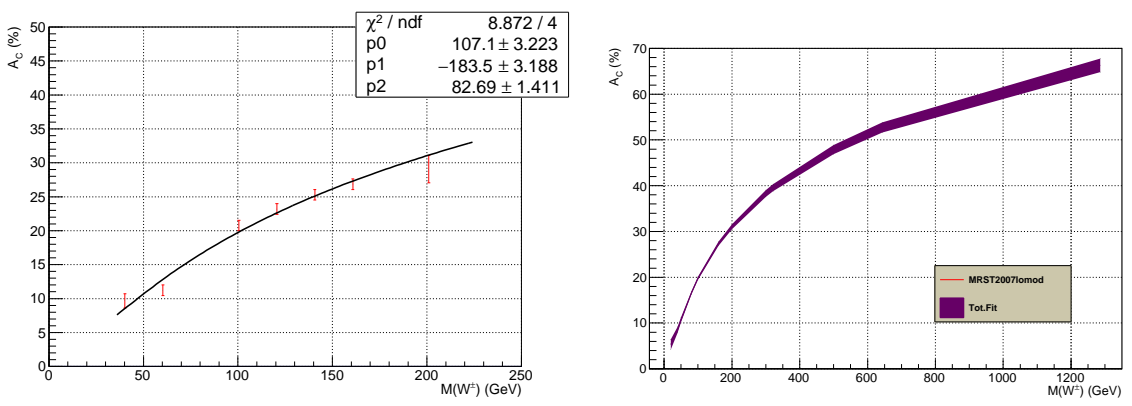

Figure 2: The $A_{C}^{\text {Meas }}$ template curve for the electron channel

\subsection{Indirect Determination of $M_{W^{ \pm}}$}

The measured $A_{C}$ from the electron and the muon channels are converted into $M_{W^{ \pm}}$estimtates using the corresponding experimental templates.

$$
\begin{aligned}
& \text { - } A_{C}^{\text {Meas.Fit }}(S)=(16.70 \pm 0.35) \% \Rightarrow M^{\text {Meas.Fit }}\left(W^{ \pm} \rightarrow e^{ \pm} v_{e}\right)=81.08_{-2.01}^{+2.06} \mathrm{GeV} \\
& \text { - } A_{C}^{\text {Meas.Fit }}(S)=(17.52 \pm 0.18) \% \Rightarrow M^{\text {Meas.Fit }}\left(W^{ \pm} \rightarrow \mu^{ \pm} v_{\mu}\right)=79.67_{-1.39}^{+3.56} \mathrm{GeV}
\end{aligned}
$$

These two indirect mass measurements are then combined through a weighted mean: $M^{\text {Comb.Meas. }}\left(W^{ \pm}\right)=80.30 \pm 0.96$ (Exp.Comb.) GeV. The theoretical template curve is read-up at 
the central value of the estimated $M_{W^{ \pm}}$and the corresponding uncertainties ${ }_{-0.21}^{+0.19} \mathrm{GeV}$ are summed in quadrature with the experimental ones so as to give the total uncertainties:

$$
M_{W^{ \pm}}=80.30_{-0.98}^{+0.98}(\text { MRST2007lomod) } \mathrm{GeV} .
$$

\section{A SUSY Physics Case}

The previous method is applied to the following SUSY "golden channel": $p+p \rightarrow \tilde{\chi}_{1}^{ \pm}+\tilde{\chi}_{2}^{0} \rightarrow$ $3 \ell^{ \pm}+\mathbb{E}_{T}$.

\subsection{Theoretical Prediction of $A_{C}\left(\tilde{\chi}_{1}^{ \pm}+\tilde{\chi}_{2}^{0}\right)$}

The theoretical $A_{C}$ template curves for the MRST2007lomod PDF. The raw template curve and the fit template curve are shown on the left hand side (LHS) and the right hand side (RHS) of Fig. 3, respectively. The fit fucntional form is analogous to that of section 2: $A_{C}\left[M_{\tilde{\chi}_{1}^{ \pm}}+M_{\tilde{\chi}_{2}^{0}}\right]=$ $\sum_{i=0}^{N} A_{i} \times\left[\log \left[\log \left[M_{\tilde{\chi}_{1}^{ \pm}}+M_{\tilde{\chi}_{2}^{0}}\right]\right]\right]^{i}$
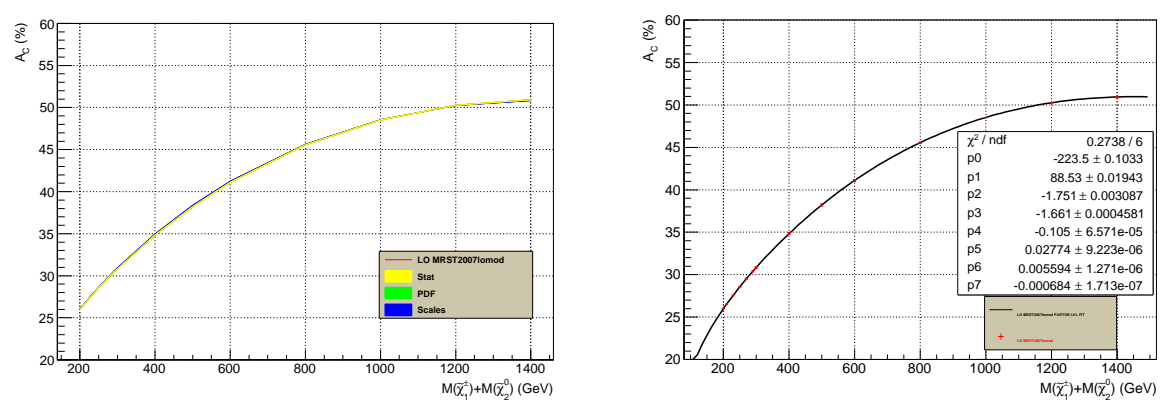

Figure 3: Theoretical $A_{C}$ template curves for the MRST2007lomod PDF.

\subsection{Experimental Measurement of $A_{C}\left(\tilde{\chi}_{1}^{ \pm}+\tilde{\chi}_{2}^{0} \rightarrow 3 \ell^{ \pm}+\mathbb{H}_{T}\right)$}

In this subsection we generate Monte Carlo samples for the signal and the background processes in $\mathrm{p}+\mathrm{p}$ colissions at $\sqrt{\mathrm{s}}=8 \mathrm{TeV}$ with an integrated luminosity of $L=20 \mathrm{fb}^{-1}$. Two different types of SUSY spectra are considered. First, there's one, denoted S1 signal, where sleptons have masses intermediate between $M_{\text {tilde } \chi_{2}^{0}}$ (set equal to $M_{\tilde{\chi}_{1}^{ \pm}}$) and $M_{\tilde{\chi}_{1}^{0}}$. Second, there's another, denoted S2 signal, where sleptons have masses much larger than $M_{\text {tilde } \chi_{2}^{0}}=M_{\tilde{\chi}_{1}^{ \pm}}$. Again, we use a fast simulation of the response of the ATLAS detector. And we apply some selection cuts to optimize the signal to noise ratio. We keep the events with three hard and isolated electron or muon that has a $p_{T}>20,10,10 \mathrm{GeV}$. These events must have a missing transverse energy larger than $35 \mathrm{GeV}$ and a stransverse mass $\left(M_{T 2}\right.$ [10][11] ) larger than $75 \mathrm{GeV}$. The $A_{C}$ background subtraction is applied, the experimental uncertainties are propagated into $A_{C}^{\text {Meas }}$ for which experimental template curves are derived. $A_{C}^{\text {Meas }}$ are converted into estimated sum of masses: $M_{\tilde{\chi}_{1}^{ \pm}}+M_{\tilde{\chi}_{2}^{0}}$ with the same treatment of the experimental and theoretical uncertainties than in section 2. 


\subsection{Indirect Determination of $M_{\tilde{\chi}_{1}^{ \pm}}+M_{\tilde{\chi}_{2}^{0}}$}

Finally, we can establish closure tests displayed in Fig. 4. The mass ranges with high signal sensitivities are highlighted.
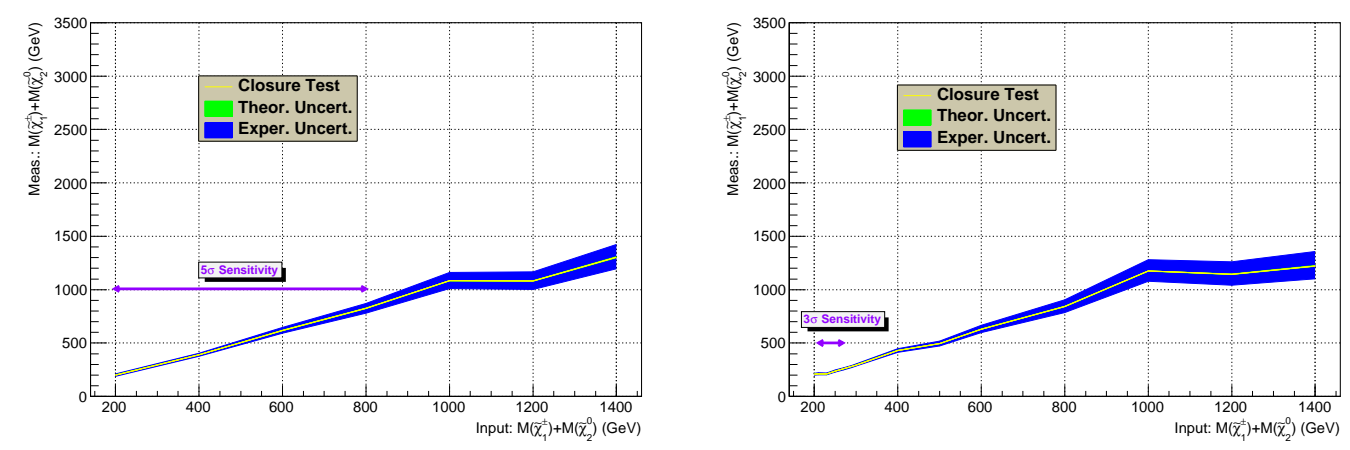

Figure 4: Closure test for the indirect measurements of $M_{\tilde{\chi}_{1}^{ \pm}}+M_{\tilde{\chi}_{2}^{0}}$ for the S1 signal (LHS) and the S2 signal (RHS)

\section{Conclusions}

We have desigend a new method that enables to extract the mass of a final state produced through charged-current production processes at the LHC. This method is independent of process the final state kinematics it essentially relies on the charge asymmetry of its initial state tehrefore on the proton PDFs. This makes this method almost model-independent. This method is especially well-suited when many final state particles escape detection. The accuracy of its indirect mass measurements are presented with three different figures of merit in Tab. 2.

\begin{tabular}{c|c|c|c}
\hline- & $W^{ \pm}$ & S1 Signal & S2 Signal \\
- & $M_{W}=80.4 \mathrm{GeV}$ & $5 \sigma:[200-800] \mathrm{GeV}$ & $3 \sigma:[210-270] \mathrm{GeV}$ \\
\hline$\frac{\delta M_{F S^{ \pm}}^{F t u}}{M_{F S^{ \pm}}^{F i t}}(\%)$ & +1.2 & {$[+3.8,+5.8]$} & {$[+3.7,+4.4]$} \\
$\frac{M_{F S^{ \pm}}^{F i t}-M_{F S^{ \pm}}^{T r u e}}{M_{F S^{ \pm}}^{T r u e}}(\%)$ & -0.1 & {$[-2.5,+3.1]$} & {$[-8.7,+1.0]$} \\
$\frac{M_{F S^{ \pm}}^{F i t}-M_{F S^{ \pm}}^{T r u e}}{\delta M_{F S^{ \pm}}^{F i t}}(\sigma)$ & -0.1 & {$[-0.7,+0.7]$} & {$[-2.4,+0.2]$} \\
\hline
\end{tabular}

Table 2: Accuracies of indirect mass measurements estimated with different figures of merit.

\section{References}

[1] G. Aad et al. [ATLAS Collaboration], Phys. Lett. B 701 (2011) 31 [arXiv:1103.2929 [hep-ex]].

[2] A. Cafarella, C. Coriano and M. Guzzi, Nucl. Phys. B 748 (2006) 253 [hep-ph/0512358]. 
[3] G. Aad et al. [ATLAS Collaboration], Phys. Lett. B 701 (2011) 31 [arXiv:1103.2929 [hep-ex]].

[4] S. Chatrchyan et al. [CMS Collaboration], Phys. Rev. Lett. 109 (2012) 111806 [arXiv:1206.2598 [hep-ex]].

[5] S. Chatrchyan et al. [CMS Collaboration], arXiv:1312.6283 [hep-ex].

[6] The LHCb Collaboration [LHCb Collaboration], "Updated measurements of $W$ and $Z$ production at $\sqrt{s}=7 \mathrm{TeV}$ with the LHCb experiment," LHCb-CONF-2011-039.

[7] [ATLAS Collaboration], ATLAS-CONF-2011-129.

[8] A. Djouadi et al. [MSSM Working Group Collaboration], hep-ph/9901246.

[9] S. Muanza, GDR SUSY Internal Note, May 2000. Unpublished. http://susy.in2p3.fr/GDR-Notes/GDR_SUSY_PUBLIC/GDR-S-076.ps.

[10] G. Polesello and D. R. Tovey, JHEP 1003 (2010) 030 [arXiv:0910.0174 [hep-ph]].

[11] D. R. Tovey, http://projects.hepforge.org/mctlib. 